\title{
Context-Awareness and Learning Fusion Method of Fluid Property Sensor Networks Based on Fuzzy Entropy
}

\author{
Zequn Zhu ${ }^{1}$, Zhuye Zhang ${ }^{2}$, Sansan Xiao ${ }^{1}$, Jiangxia Zou ${ }^{3}$, Xinyu Hou ${ }^{3}$, Zhengying Cai ${ }^{1, ~ *}$ \\ ${ }^{1}$ College of Computer and Information Technology, China Three Gorges University, Yichang, China \\ ${ }^{2}$ School of Law and Public Administration, China Three Gorges University, Yichang, China \\ ${ }^{3}$ School of Foreign Languages, China Three Gorges University, Yichang, China
}

\section{Email address:}

1152966090@qq.com (Zequn Zhu),2721089415@qq.com (Zhuye Zhang), yishikongmi@qq.com (Sansan Xiao), 785959370@qq.com (Jiangxia Zou),376026330@qq.com (Xinyu Hou), master_cai@163.com (Zhengying Cai)

${ }^{*}$ Corresponding author

\section{To cite this article:}

Zequn Zhu, Zhuye Zhang, Sansan Xiao, Jiangxia Zou, Xinyu Hou, Zhengying Cai. Context-Awareness and Learning Fusion Method of Fluid Property Sensor Networks Based on Fuzzy Entropy. American Journal of Networks and Communications. Vol. 5, No. 6, 2016 , pp. 128-138. doi: 10.11648/j.ajnc.20160506.12

Received: October 24, 2016; Accepted: November 7, 2016; Published: December 26, 2016

\begin{abstract}
Fluid sensor network is very difficult to make context-awareness and learning fusion because there is a variety of complex dynamic uncertainties involved ranging from information redundancy, information complementary, to information instability. This paper introduces a fuzzy entropy method into context-awareness and learning fusion method of fluid property sensor networks. First, the architecture of fluid property sensor network is analyzed, and based on it the context characteristics are described. Second, by the introduction of fuzzy entropy, the learning fusion method of fluid property sensor networks is proposed, where the fusion hierarchy of context information is discussed and the fusion algorithm is also illustrated. Third, an example is presented for verification of the proposed model, where the multiple sensor information fusion based on fuzzy logic analysis method can effectively tackle uncertain information. At last, some interesting conclusions are carried out and future researching directions are also indicated at the end of the paper.
\end{abstract}

Keywords: Fluid Properties Sensor, Context Awareness, Learning Fusion, Fuzzy Entropy, Uncertain Information

\section{Introduction}

Today, fluid property sensor is very popular because it provides us a cheap and convenient tool to monitor the fluid property. Afkhami (2014) illustrated a sensitive electrochemical sensor for rapid determination of methadone in biological fluids using carbon paste electrode modified with gold nanofilm [25]. Schmitt (2014) developed a microthermal sensors for determining fluid composition and flow rate in fluidic systems [22]. Li (2014) talked about novel magnetic field sensor based on magnetic fluids infiltrated dual-core photonic crystal fibers [12]. Dey (2015) proposed a VO2 nanorods for efficient performance in thermal fluids and sensors [2]. Xie (2015) modeled a kind of parameters optimization of magnetic fluid micropressure sensor [10]. Pajares (2015) put forward a kind of sensors for fluid leak detection [1]. He (2015) brought forward a self-healing electronic sensor based on thermalsensitive fluids [3]. What's more, Amrehn (2015) introduced a photonic crystal-based fluid sensors in practical application [5]. Schultz (2015) suggested a lateralmode vibration of microcantilever-based sensors in viscous fluids using timoshenko beam theory [6]. Bley (2016) indicated a degradation monitoring of aviation hydraulic fluids using non-dispersive infrared sensor systems [15].

More fluid sensors were integrated to form a sensor networks to get more comprehensive information about fluid property. Yadav (2014) studied a biocompatible nano gold modified palladium sensor for determination of dopamine in biological fluids [17]. Alarfaj (2014) made a model of construction and validation of new electrochemical carbon nanotubes sensors for determination of acebutolol hydrochloride in pharmaceuticals and biological fluids [21]. Kulapina (2015) built a 
potentiometric sensors for the determination of some cephalosporin antibiotics in biological fluids and medicinal preparations [7]. Pattar (2015) described polybenzoin based sensor for determination of 2thiouracil in biological fluids and pharmaceutical formulations [18]. Gupta (2015) considered a sensitive polymelamine sensor for the determination of lomefloxacin in biological fluids [19]. Couto (2015) made a research of screen-printed electrode based electrochemical sensor for the detection of isoniazid in pharmaceutical formulations and biological fluids [23]. Tadi (2016) extended the voltammetric determination of pindolol in biological fluids using molecularly imprinted polymer based biomimetic sensor [16]. Raj (2016) concerned poly-melamine film modified sensor for the sensitive and selective determination of propranolol, with a beta-blocker in biological fluids [24]. Some sensors are supposed to monitor biological fluids.

A multi-sensor networks can provide us a capability of context awareness. Matzeu (2015) summarized the advances in wearable chemical sensor design for monitoring biological fluids [4]. Fernandes (2016) offered an osmotic pressure sensor for monitoring the level of hydration in biological fluids [14]. Sensors can also be used in other ways. Kamel (2014) considered a MIP-based biomimetic sensors for static and hydrodynamic potentiometric transduction of sitagliptin in biological fluids [8]. Stefan-van (2014) evaluated the multimode sensors based on nanostructured materials for simultaneous screening of biological fluids for specific breast cancer and hepatitis B biomarkers [20]. Varma (2015) researched a cell-based sensor of fluid shear stress for microfluidics [11]. Electrochemical analysis sometimes will be used in sensors. Ali (2014) implied a fluid interface-mediated nanoparticle membrane as an electrochemical sensor [9]. Rosy (2014) reviewed graphene modified palladium sensor for electrochemical analysis of norepinephrine in pharmaceuticals and biological fluids [13].

However there are many uncertainties in fluid property sensor networks, so it is very difficult to make the context- awareness and learning fusion method. This paper introduces a fuzzy entropy method into context-awareness and learning fusion method of fluid property sensor networks. First, the architecture of fluid property sensor network is analyzed, and based on it the context characteristics are described. Second, by the introduction of fuzzy entropy, the learning fusion method of fluid property sensor networks is proposed, where the fusion hierarchy of context information is discussed and the fusion algorithm is also illustrated. Third, an example is presented for verification of the proposed model, where the multiple sensor information fusion based on fuzzy logic analysis method can effectively tackle uncertain information to understand the various uncertainties in working with unknown fluid properties and to find solutions to these problems. At last, some interesting conclusions are carried out and future researching directions are also indicated at the end of the paper.

\section{Context Awareness of Fluid Sensor Network}

\subsection{Architecture of Fluid Property Sensor Network}

By connecting to the fluid nature of the sensor system, the flow tube, can detect the corresponding part of the fluid. This is covered with wireless sensor grid work fluid properties of the sensor, used to get detailed information of monitored fluid, as shown in fig. 1. Quick sort of wireless sensor network provides a type of information processing, namely receiving a variety of information fusion, and process analysis by fluid property sensor networks, and the next stage of the learning fusion task can learn by a fusion. Therefore, the learning fusion task is carried out dynamically. The specific learning fusion types ensure which specific one from a family of context awareness rules to be activated to bring the part's present location to the aim in the workspace containing fluid.

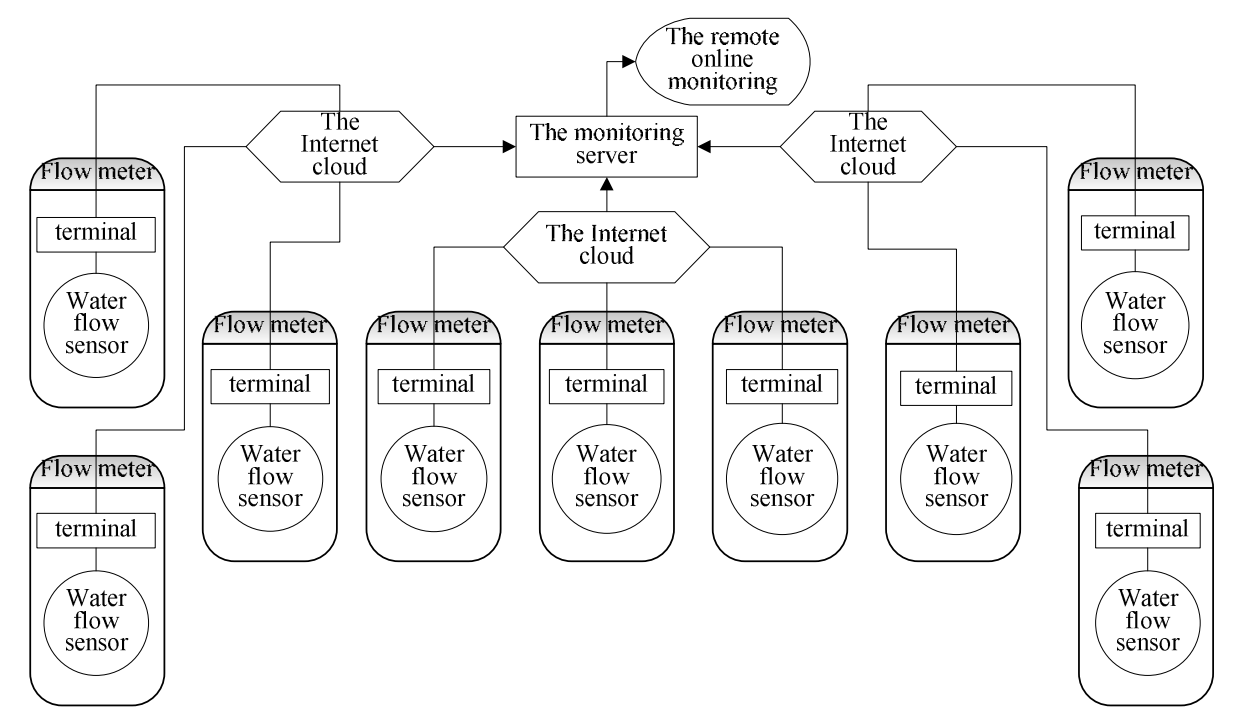

Fig. 1. A typical fluid sensor networks. 
Wireless sensor network can be divided into roughly, the physical layer, data link layer, network layer, transport layer and application layer. The physical layer is the computer network the lowest layer of the OSI model.

The physical layer provides us a tool to preach. Losing data needed physical link to create, maintain, dismantle, and provide with mechanical, electronic, functional features and specifications. In short, the physical layer is to ensure that the original data. In the various physical media transmission. $\left\{a_{i} \mid i=1, \ldots, 54\right\}$, wireless network mode of value is 1 to enter part of the module located in the region, otherwise, its value is $0,(i=1, \ldots, 54)$. The last one and 55 of input nodes contain the internal target information as a cube or cuneate, if the target is a rectangular profile, the value of 55 input nodes is 1 or 0 .

The physical layer of the wireless sensor network is in the OSI layer 1, although at the bottom it is the basis for the rest of the open system. Providing a physical transmission medium for data communication between devices and interconnecting devices, it is a reliable environment for data transmission. If you want to use this word to remember the first layer as little as possible, this is signal and medium.

According to the network topology structure of the neural network, classifiers can be divided into two categories: hierarchical structure and network structure. The hierarchical structure of the neural network will be divided into output neuron function and sequence layer, interface layer, hidden layer and output layer. The neurons in output layer are responsible for receiving input information from the outside world, and each hidden layer neuron. The inner layer of the hidden layer neural network is responsible for the information transformation. According to the need to design a layer or layer; the last hidden layer output layer neurons transfer information to the outside world after further processing information of output results. Type and interconnection network structure, may be connected between any two nodes of the path, so according to the degree of network connectivity to the nodes, the interconnection network is divided into three conditions: full interconnection, local interconnect and sparse connections type.

If the target of the mesh on a straight line is a triangle top view and a cube, the desired output is 1 , otherwise it is 0 . It is possible that different input patterns from 55 inputs are used to identify an learning fusion type. Six sets of sample input modes are selected for training purposes, or for each possible outcome. It is important to note here that the neural training mode has fed all possible different input data into one of six outputs.

\subsection{Context Characteristics Analysis}

Assuming that the workspace portion is in an unknown fluid environment, that is, the working environment is unknown, the inside environment block is unknown but close to the sensor's fluid properties. The macro learning fusing tasks is to perform the sensor's fluid properties rather than the visual sensor, because the depth information of the target and the visual flow of the sensor inside, and these factors can not communicate directly with the other sensors.

The fluid properties of the moving linear mesh sensor target information are accurate, such as velocity and direction of the flow and pressure of the internal target. The goal of the speed and direction of the relevant information is to detect various properties of the fluid. A plurality of fluid properties sensors was provided to reduce the time required to acquire the target information and to attach the track pad to the movable and free directions.

The $x, y, z$, directional centers $\left(x_{c}, y_{c}, z_{c}, \theta_{c}\right)$ of $\theta$, and the inside shapes of monitored targets that should be located in that over targets can be given.

$$
\begin{aligned}
& h_{x(\text { center })}=l_{2} \cos \theta_{h}+l_{1} \sin \theta_{h}+a_{x_{2}} \\
& h_{y(\text { center })}=l_{4} \sin \theta_{h}+l_{3} \sin \theta_{h}+a_{y_{4}}
\end{aligned}
$$

where the tilt angle $\theta_{h}$ of target is given as

$$
\tan ^{-1}\left[\left(\left|a_{y_{1}}-a_{y_{2}}\right|\right) /\left(\left|a_{x_{1}}-a_{x_{2}}\right|\right)\right] .
$$

The lengths in (1) are

$$
\begin{gathered}
I_{1}=\frac{\sqrt{\left(a_{y_{4}}-a_{y_{2}}\right)^{2}+\left(a_{x_{4}}-a_{x_{2}}\right)^{2}}}{2} \\
I_{2}=\frac{\sqrt{\left[\left(a_{x_{2}}-a_{x_{1}}\right)+\left(l_{1}-l_{5}\right) \sin \theta_{h}\right]^{2}+\left[\left(a_{y_{2}}-a_{y_{1}}\right)+\left(l_{5}-l_{1}\right) \cos \theta_{h}\right]^{2}}}{2} \\
I_{3}=\frac{\sqrt{\left(a_{y_{4}}-a_{y_{3}}\right)^{2}+\left(a_{x_{4}}-a_{x_{3}}\right)^{2}}}{2}
\end{gathered}
$$

The center position is

$$
\begin{aligned}
& D_{x(\text { center })}=l_{1} \cos \theta_{h^{\prime}}+a_{x_{2}}, \\
& D_{y(\text { center })}=l_{1} \sin \theta_{h^{\prime}}+a_{y_{2}}
\end{aligned}
$$

where the tilt angle is

$\theta_{h}=\tan ^{-1}\left[\left(\left|a_{y_{3}}-a_{y_{2}}\right|\right) /\left(\left|a_{x_{3}}-a_{x_{2}}\right|\right)\right]$ and $\theta_{h^{\prime}}=\theta_{h}+30^{\circ}$.

The length in (2) is $I_{1}=\sqrt{\frac{\left(a_{y_{2}}-a_{y_{1}}\right)^{2}+\left(a_{x_{2}}-a_{x_{1}}\right)^{2}}{3}}$.

Assuming that at time $t=t_{i}(i=1, \ldots, n), a\left(t_{i}\right)$ stands for the position of $B_{o p}$, and $\left(a_{x_{i}}, a_{y_{i}}\right)$ is the coordinate of $\mathrm{x}$ and $\mathrm{y}$ for $a\left(t_{i}\right)$ in accordance with $\left(x_{w}, y_{w}, z_{w}, \theta_{w}\right)$.

The center location $\mathrm{x}$ and $\mathrm{y}$ of circle target is given as

$$
\begin{gathered}
D_{x(\text { center })}=\frac{\sqrt{\left(a_{x_{3}}-a_{x_{2}}\right)^{2}+\left(a_{y_{3}}-a_{y_{2}}\right)^{2}}}{2}+a_{x_{2}} \\
D_{x(\text { center })}=\frac{\sqrt{\left(a_{x_{3}}-a_{x_{2}}\right)^{2}+\left(a_{y_{3}}-a_{y_{2}}\right)^{2}}}{2}+a_{x_{2}}
\end{gathered}
$$

The context awareness information obtained from fused 
sensor networks can be expressed as the parts of target component, and can be sent by the fusion process of a context learning.

\section{Learning Fusion Method Based on Context Awareness}

\subsection{Learning Fusion Method of Fluid Property Sensor}

In order to solve the uncertainty problem associated with the wireless sensor network procedure, fuzzy set theory is built to make the procedure be optimized using appropriate cost functions. The lower the probability of the input value of the wireless sensor network is identified with the desired output, the higher the degree of the uncertainty is. A degree of identification between the input pattern and the desired output of the wireless sensor network can be measured by means of the fuzzy entropy.

Learning fusion system's task is to generate an appropriate control action to the position of the part into the target location fusion of learning. As a process control, it uses the angle and the diagonal movement as the main direction to become an auxiliary movement and as the direction of $X$ and $Y$. According to the observation of the environment conditions that the part faced, the specific rule base is activated, and in such a manner the controlled part can avoid fluid and keep the situation. There are some assumptions as follows.

First, the fluid flow in three cases takes place in this turn: to the left in front, to the front and right, and to the right and left parts of the present position.

Second, the fluid is all placed at a higher point than the lowest one.

Third, a vision sensor is placed in the workspace to detect every object in the whole fluid workspace by a twodimensional viewpoint.

Finally, the part attached on the fluid property sensor networks has its own fixed coordinates and the coordinate relative to the world coordinate moving direction by fuzzy functions shown in Fig. 2.

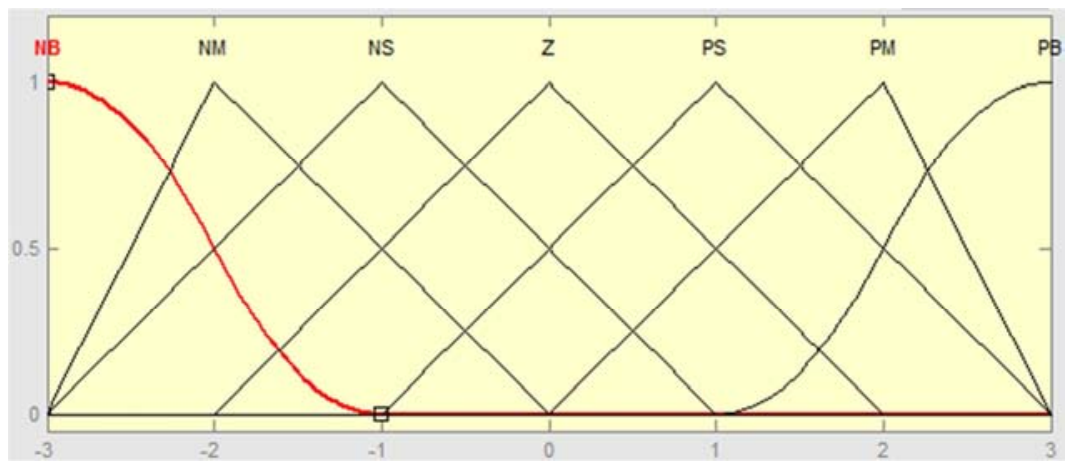

Fig. 2. Fuzzy membership functions.

The variables in Fig. 2 represent that $d_{t}$ is the diagonal distance where $d_{p}$ and $\theta_{p}$ are produced by learning, the fusion part of the control parameters, and the diagonal angle moving to the target position, respectively. $G_{l}$ and $G_{r}$ are the center of the distance along the $\mathrm{X}$ axis, respectively, to the leftmost and the rightmost point in the direction of the part of the fluid. $d_{h}$ is the sum of the distances $d_{p w}$ and $G_{r}$ (or $G_{l}$ ), where $d_{p w}=d_{p w}^{\prime}+\alpha$ and $d_{p w}^{\prime}$ with a safety margin $\alpha . d_{f}$ is the distance. $x_{d_{h}}$ and $y_{d_{f}}$ is part of vertical and horizontal movement of the relative distance part of the process generated by the fusion study $d_{h}$ and $d_{f}$, respectively, in order to avoid obstacles toward the target. $G_{h}$ and $G_{b}$ along the center of the part at present, are the highs and lows with the distance of the $Y$ axis respectively. $d_{v}$ is the sum of the distances of $d_{p l}$ and $G_{h}$ ( or $G_{b}$ ), namely $d_{p l}=d^{\prime}{ }_{p l}+\beta . d_{1 r}$. It is the distance between the center part of the $X$ axis and the obstruction at the far left or right. $x_{d_{1 r}}$ and $y_{d_{v}}$ are the sensor's horizontal movement and vertical movement related to the distances $d_{1 r}$ and $d_{v}$, respectively. A part of learning fusion is used to avoid the obstacle to the object, and the part of the optimal path generation control which is measured by the fuzzy entropy is defined as the value, $\theta_{p_{i}}, d_{p_{i}}, x_{d_{h_{i}}}, y_{d_{f_{i}}}$, $x_{d_{1 r_{i}}}$, and $y_{d_{v_{i}}}(i=1,2, \ldots, n)$.

In each step, the topological relationship is used to determine a specific rule base between the object and the target, which is used to find the set of feasible paths. The sum of rules which is based on variables and used to study the process of fusion includes $\theta_{t}, d_{t}, \theta_{p}, d_{p}, d_{h}, d_{f}, x_{d_{h}}$, $y_{d_{f}}, d_{1 r}, d_{v}, x_{d_{1 r}}$, and $y_{d_{v}}$.

The method of rule base is as follows. If the part which is kept is moving towards a target with the control parameters $x_{d_{h}}, y_{d_{f}}, x_{d_{1 r}}$, and created by the learning fusion according to the following three basis in Table 1 .

If the obstacle moves to a target which is located in $\theta_{t} \leq 90^{\circ}$, the first move in the $+y$ direction is $d_{v}\left(=d_{p_{l}}+G_{h}\right)$, then moved to the $+x$ direction for distance $d_{1 r}$, and finally the target position can be gotten in Fig. 2 . This rule stipulate that if $d_{1 r}$ is medium small, and $d_{v}$ is small zero, then $x_{d_{l h}}$ should be medium small and small zero 
for $y_{d_{v}}$.

If the obstacle's part is in $\theta_{t}>90^{\circ}$, the distance is $d_{v}\left(=d_{p_{l}}+G_{h}\right)$ in the $+y$ direction, then the distance $d_{1 r}$ moves in the $-x$ direction, and at last to the target position.

If the obstacle is based upon $\theta_{t}$ : first, if $\theta_{t} \leq 90^{\circ}$, the first move in the $+x$ direction is $d_{h}\left(=d_{p_{w}}+G_{r}\right)$, then the distance is $d_{f}$ in the $+y$ direction, and lastly moves to the target position. Second, if $\theta_{t}>90^{\circ}$, the rule base for this situation is similarly gained.

Table 1. Rulebase for obstacle located to the right and $y_{t} p 90$.

\begin{tabular}{llllllll}
\hline $\boldsymbol{d}_{1 \boldsymbol{r}}$ & $\boldsymbol{d}_{\boldsymbol{v}}$ & $\boldsymbol{x}_{1 \boldsymbol{r}}$ & $\boldsymbol{y d}_{\boldsymbol{v}}$ & $\boldsymbol{d}_{1 \boldsymbol{r}}$ & $\boldsymbol{d}_{\boldsymbol{v}}$ & $\boldsymbol{x d}_{1 \boldsymbol{r}}$ & $\boldsymbol{y d}_{\boldsymbol{v}}$ \\
\hline MS & MD & MS & MD & $\ldots$ & $\ldots$ & $\ldots$ & $\ldots$ \\
MS & VL & MS & VL & VL & MD & VL & MD \\
MS & SZ & MS & SZ & VL & VL & VL & VL \\
MS & LR & MS & LR & VL & SZ & VL & SZ \\
MS & MS & MS & MS & VL & LR & VL & LR \\
SZ & MD & SZ & MD & VL & MS & VL & MS \\
SZ & VL & SZ & VL & LR & MD & LR & MD \\
SZ & SZ & SZ & SZ & LR & VL & LR & VL \\
SZ & LR & SZ & LR & LR & SZ & LR & SZ \\
SZ & MS & SZ & MS & LR & LR & LR & LR \\
MD & MD & MD & MD & LR & MS & LR & MS \\
\hline
\end{tabular}

By using the property of inequality, it can be used as an inference method to find a set of fuzzy variables. The inequality is used to infer the fuzzy relations with the minimal inference engine.

The learning fusion provides a suitable point for setting a low level of fluid sensor network, mainly including task execution and torque calculation techniques. For instance, the distance between the angle and the diagonal can control the low level of sensors related to other parameters. The torque variables are $(\Delta f, \Delta \tau)$ for each link by means of its own control program.

\subsection{Learning Fusion Method with Fuzzy Entropy}

The uncertainty is known as a function of fuzziness and fuzzy sets is called fuzziness. An action assigns a real value $f(Y)$ to each subset $Y \in W$ which shows a degree of fuzziness. The membership value used to measure the uncertainty associated with the fractional extraction process is then obtained from the membership function based on the finite precision sensor information formulation. The following mean (4) or (5) is used to measure the extent of uncertainty connected with a feasible plan. The entropy $f$ of $Y=\left\{x, \mu_{Y}(x) \mid x \in W\right\}$ is defined as $f(Y)=\eta \sum_{i=1}^{n} \phi\left[\mu_{Y}\left(x_{i}\right)\right]$.

The total entropy $p_{s}$ is

$$
E\left(p_{s}\right)=\eta \sum_{k=1}^{n} \sum_{l=1}^{m} \phi_{k l}\left[\mu_{Y}\left(x_{l}\right)\right]
$$

where $\mu_{Y}(x)$ is the membership function of $\mathrm{x}$ in $\mathrm{W}, \eta$ is a constant, and $\phi_{k l}(x)=-x \ln x-(1-x) \ln (1-x)$. Fig. 2 reveals the fuzzy entropy measure of control parameter $e_{\text {control }}$ with the sum of attribute $\phi\left(N_{g_{i}}\right)$ to the membership grades $N_{g_{i}}(i=1,2,3)$.

The Euclidean distance with $\beta=2$ can also be measured by $p_{s}$.

$$
E\left(p_{s}\right)=\sum_{k=1}^{n}\left[\sum_{l=1}^{m}\left|\mu_{Y}\left(x_{l}\right)-\mu_{C}\left(x_{l}\right)\right|^{\beta}\right]^{1 / \beta}, x \in W,
$$

where $\mu_{C}(x)$ has a specific crisp value in $\mu_{Y}(x)$, and $\beta \in[1, \infty]$.

There are many reasons for the uncertainties associated with the methods described in the fluid sensor networks. The fact that multiple viable paths can be generated with a different order of movement, is also a less important reason of uncertainty. In a feasible plan, a plan with the lowest degree of uncertainty in the fuzzy entropy measure (4) or (5) is selected as a plan for performing a specific task. With nearoptimal selection, the uncertainty associated with the fuzzy set of plans is significantly reduced.

\section{Optimization Function of Fluid Property Sensor Network}

There are uncertainties in the results related to the implementation of wireless network tasks. The uncertainty of this result is due to the uncertainties, incompleteness and inaccuracy of the measurements made by the sensors and the uncertainty of the multi-sensor information fusion. For example, the relevant information for the input neural network is from a sensor system, such as a fluid property sensor system with finite precision. In addition, all the information received is directly related to determining the control parameters associated with the type of component and the movement of the fluid property sensor networks. The fuzzy set theory was introduced to measure the uncertainty associated with the task execution of the macro components. In this method, the cost function with a minimum fuzzy entropy, can be used to optimize the process. The correlation between the learning and fuzzy entropy of a fluid sensor network is given by the following formula.

The value of the probability is the upper limit of the probability. That is,

$$
\pi(x) \geq p(x)
$$

Although the correlation between probabilities and possibilities is relatively weak, this relative relationship still exists and is generally heuristic rather than the definitive principle that can be used to accurately compute the results. It is called the principle of consistency between probability and probability [14]. Supposing that the value of variable $\psi$ is a fuzzy set, and all possible values of $\psi$ are contained in the universe of discourse $W$, the fuzzy restriction in $W$ 
determines fuzzy set Y. So the probability distribution $\pi_{\psi}$ defines the ambiguity limit of the variable $\psi$ in $W$ as its value. $\pi_{\psi}(x)$ represents the possibility of $\psi=x$. These $\pi_{\psi}(x)$ are form a fuzzy set $Y$. Thus, the value of the probability distribution function $\pi_{\psi}(x)$ agrees with the membership degree $\mu_{Y}(x)$ of the fuzzy set $Y$. That is,

$$
\mu_{Y}(x)=\pi_{\psi}(x), x \in W .
$$

Assuming that an arbitrary fuzzy set $Y$ is a function of a sample space $\Omega(Y)$ on $[0,1]$ and $Y$ is a fuzzy group in the Euclidean space $B^{n}$ where the subscript $n$ in $B^{n}$ represents the number of element, namely, $\underline{Y}=\left\{Y_{i} \mid i \in(1,2, \ldots)\right\}$. The probability of the fuzzy number $Y \in \underline{Y}$ is a fuzzy set which is defined in the space $B^{n}$. A fuzzy probability estimate $P(Y)$ is a mapping of $Y$ on $[0,1]$. The fuzzy set $Y \in B^{n}$ can be expressed as

$$
Y=\left\{\left(x, \mu_{Y}(x)\right) \mid x \in B^{n}\right\}
$$

Based on the above concept, the neural network $G_{k}^{\varsigma}$ is normalized by

$$
\varphi_{k}^{a_{\varsigma}}=\frac{G_{k}^{\varsigma}}{G_{k}^{d}}
$$

where $\varphi_{k}^{a_{\varsigma}}(k=1,2, \ldots, r)$, is the normalized output of node $k$ at the $\varsigma$ th. $G_{k}^{\varsigma}$ is the output of the $k$ th output node at the $\varsigma$ th. $G_{k}^{d}$ is the desired output of the $k$ th node.

Since the input pattern of fluid sensor networks is obtained by a sensor system with limited accuracy, there is uncertainty. So the normalized output of the neural network for each training step can be written as the fuzzy set $Y$ in (8). In addition, the normalized actual output value $\varphi_{k}^{a_{\zeta}}$ of each output node of the neural network at a particular training step $\varsigma$ may be considered as a possibility according to (6), (7) and the above-mentioned principle of probability and probability coincidence. Finally, the probability of the fuzzy set $F$ in (8) at training period $\varsigma$ is obtained by

$$
P\left(Y_{\zeta}\right)=\frac{\sigma}{\sum_{k=1}^{r} e^{\left\|\Phi_{d}^{h}\right\|}}\left(=\frac{\sigma}{\sum_{k=1}^{r} e^{\left\|\Phi_{k}^{d_{\zeta}}-\Phi_{k}^{a_{\zeta}}\right\|}}\right)
$$

The condition (11) indicates the probability that the input pattern is successfully recognized as the desired value output in the specific training step $\zeta$. In (11), $\left\|\Phi_{d}^{h}\right\|$ is the Hamming distance, $\Phi_{k}^{d_{\zeta}}$ is the normalized excepted output of the kth node at the $\varsigma$ th, $\Phi_{k}^{a_{\zeta}}$ is described in (9), and $\sigma$ is a scaling factor and is usually set equal to $r$.

As an untrained input mode with outcome $\varphi_{6}^{d}$, the fuzzy set $Y$ at the epoch of 240 can be described as

$$
P\left(Y_{240}\right)=\frac{\sigma}{\sum_{k=1}^{6} e^{\left\|\Phi_{k}^{d_{240}}-\Phi_{k}^{a_{240}}\right\|}}
$$

The excepted output at the training step is 240. As the probability $P\left(Y_{\varsigma}\right)$ of a fuzzy set $Y_{\varsigma}$, it can be converges to the normalized expected output $\Phi_{6}^{d}$.

\section{Experiment and Analysis}

\subsection{Problem Description}

In order to quantify the content discussed in the previous section to a certain extent, the proposed method is applicable to some unknown working environment space. The purpose of the context-awareness and learning fusion method is to move the components in the corners, diagonals and $x$ and $y$ directions corresponding to the event, in spite of the fluid obstruction, bringing the components near the target for the purpose of the next operation, $e_{\theta_{p_{i}}} ; e_{d_{p_{i}}} ; e_{x_{d_{h_{i}}}} ; e_{y_{d_{f_{i}}}} ; e_{x_{d_{l_{\gamma_{i}}}}}$, and $e_{y_{d_{v_{i}}}}(i=1,2, \ldots, n)$ through the algorithm in Section 3.1

The four sides of the rectilinear grid are $(0,0),(0,1),(1,0)$, and $(1,1)$, respectively. The sizes of the aims of learning fusion are as the follows. The size of the cube target can be set to $3.2 * 2.1 \mathrm{~cm}^{2}$, and the radius of the circular-shaped target is measured as $0.5 \mathrm{~cm}$.

Here, the starting position and the target position keep fixed, and the target is $102.4 * 86.4 \mathrm{~cm}^{2}$. The starting position of the part and the center of the target are $(0,0)$ and $(51.2$, 43.2), respectively. The border of the five fluid are shown in Table 2 .

Table 2. The initial parameters.

\begin{tabular}{lllll}
\hline$G_{a}$ & $(4.5,15.6)$ & $(4.4,19.8)$ & $(8.6,15.6)$ & $(8.6,19.8)$ \\
$G_{b}$ & $(8.3,26.0)$ & $(9.1,31.2)$ & $(12.5,26.0)$ & $(13.4,31.2)$ \\
$G_{c}$ & $(11.8,12.5)$ & $(11.7,16.7)$ & $(16.8,12.5)$ & $(16.8,16.7)$ \\
$G_{d}$ & $(21.3,15.6)$ & $(21.4,19.8)$ & $(25.5,15.6)$ & $(25.5,19.8)$ \\
$G_{e}$ & $(23.7,6.3)$ & $(25.2,10.4)$ & $(29.9,6.3)$ & $(30.3,10.4)$ \\
\hline
\end{tabular}

The offset control parameters of the part's movements are obtained by

$$
\mu_{Y}(x)=\frac{1}{\lambda}-(-|x-\sigma|+\lambda) \vee 0(\lambda>0)
$$

The lower the fuzzy entropy is, the higher the probability of success gets. The variable $p_{a}$ represents the path generated by applying learning fusion in section 3.1 and $p_{b}$ represents the path generated by applying another learning fusion with different rule bases in section 3.1. The constants $\lambda$ and $\sigma$ are adjusted in the light of the linguistic terms. The function (13) related to the value $x_{d_{1} \text { r }}$ for the obstacle to the left of the part with $\theta_{t}>90^{\circ}$. The linguistic variables for fuzzy input and output are $\theta_{t}, d_{t}, \theta_{p}$, and fuzzy language set is $\{\mathrm{NB}, \mathrm{NM}, \mathrm{NS}, \mathrm{Z}, \mathrm{PS}, \mathrm{PM}, \mathrm{PB}\}$, for the obstacle located 
in front of the part and $\theta_{t} \leq 90^{\circ}$. The fuzzy rules are shown in Fig. 3.

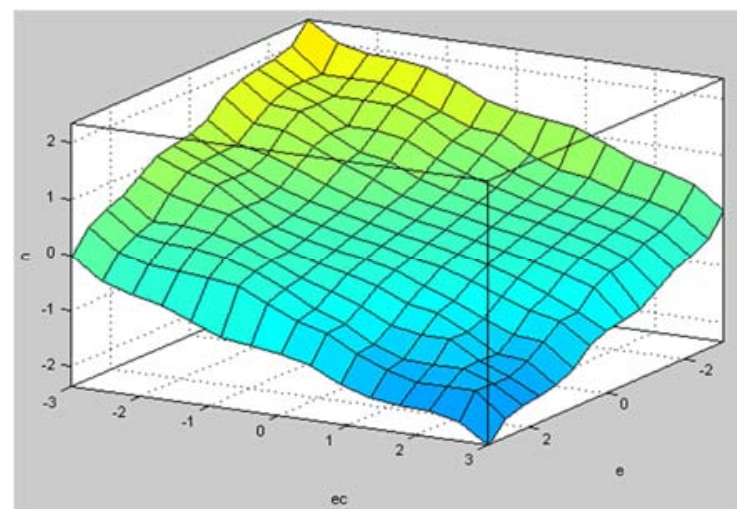

Fig. 3. Fuzzy rules.

By the fuzzy rules, the sensor networks can learn from each other based on context awareness information.

\subsection{Result Analysis}

In this section, a macro component with a learning and sensor fusion method for taking a part to the target location was introducing. By combining the fluid property sensor data, the characteristics of the tasks associated with the learning action process are identified. The learning method is used to reduce the uncertainty associated with partially introduced task execution. The probability of success and fuzzy entropy are shown in fig 4 and 5, respectively.

As you can see, high probability of success mean the proposed model can be applied to different sets of fuzzy rule bases in the same operating environment. The cost function of the optimal learning fusion is determined by The uncertaintyassociated with the path. That is, the optimal path is the one with the lowest uncertainty in fuzzy entropy. As the uncertainties of the feasible paths $p_{a}$ and $p_{b}$, The plan $p_{a}$ is divided into six stages.

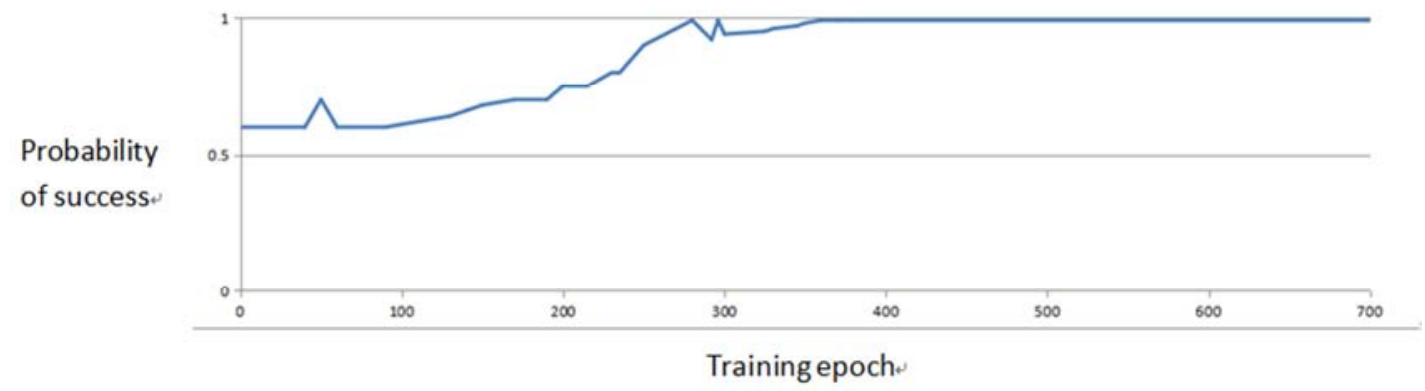

Fig. 4. Probability of success.

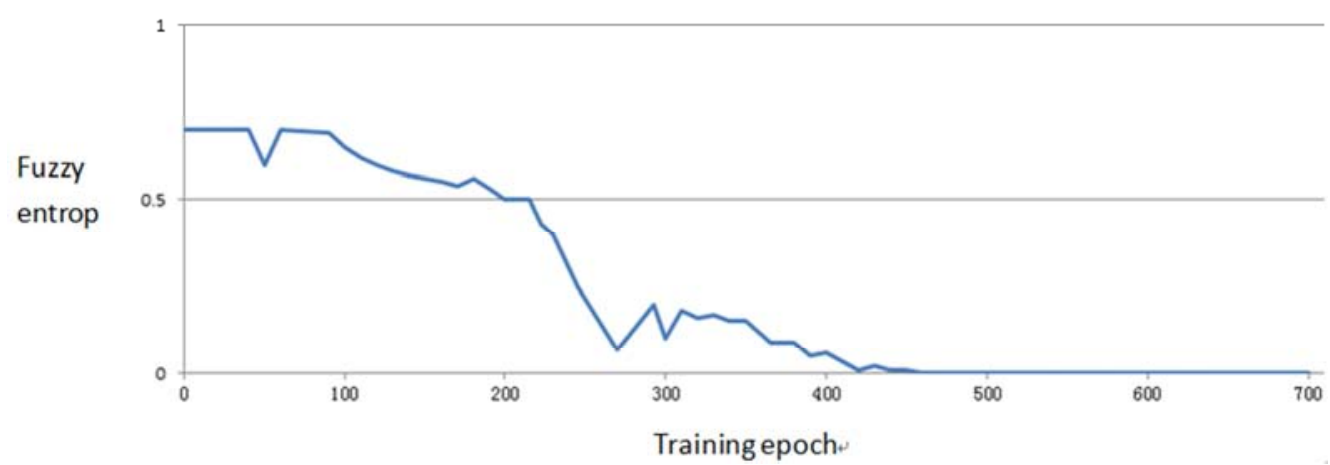

Fig. 5. Fuzzy entropy.

The entropy of the viable paths other than the paths $p_{a}$ and $p_{b}$ are measured as paths higher than $p_{a}$ and $p_{b}$, and thus are omitted. the path $p_{a}$ has the lowest degree of uncertainty, and the fuzzy entropy measure is with the highest probability of 0.976 , so $p_{a}$ is selected as the best path for the execution of that particular task.

The entropy path is obtained as follows. Path $p_{a}$ includes the control parameters of six phases. As shown in Fig. 2, the total entropy of the path $p_{a}$ is the sum of the entropy of each control parameter by measuring the entropy of each control parameter one by one. While the total entropy of path $p_{b}$ is 13.6343. In the learning fusion in Section 3.1 and in another coordinator with different rule bases, the path $p_{a}$ generated by the coordinator in Section 3.1 is optimal.

For the direction of fluid resistance in front of the component, $\theta_{t}>90^{\circ}, d_{f}, y_{d_{f}}, d_{v}, y_{d_{v}}=\{\mathrm{NB}, \mathrm{NM}, \mathrm{NS}, \mathrm{Z}$, PS, PM, PB $\}, \mathrm{d}_{1 \mathrm{r}}$ and $\mathrm{x}_{\mathrm{d}_{\mathrm{lr}}}=\{\mathrm{NB}, \mathrm{NM}, \mathrm{NS}, \mathrm{Z}, \mathrm{PS}, \mathrm{PM}, \mathrm{PB}\}$ for the fluid resistance located to the left of the part and $\theta_{t}>90^{\circ}$, and $\mathrm{d}_{1 \mathrm{r}}$ and $\mathrm{x}_{\mathrm{d}_{\mathrm{lr}}}=\{\mathrm{NB}, \mathrm{NM}, \mathrm{NS}, \mathrm{Z}, \mathrm{PS}, \mathrm{PM}, \mathrm{PB}\}$ for the fluid resistance located to the right of the part and 
$\theta_{t} \leq 90^{\circ}$

According to the learning fusion in Section 3.1 and in another coordinator with different rule bases, the sumsquared error is shown in Fig. 6.

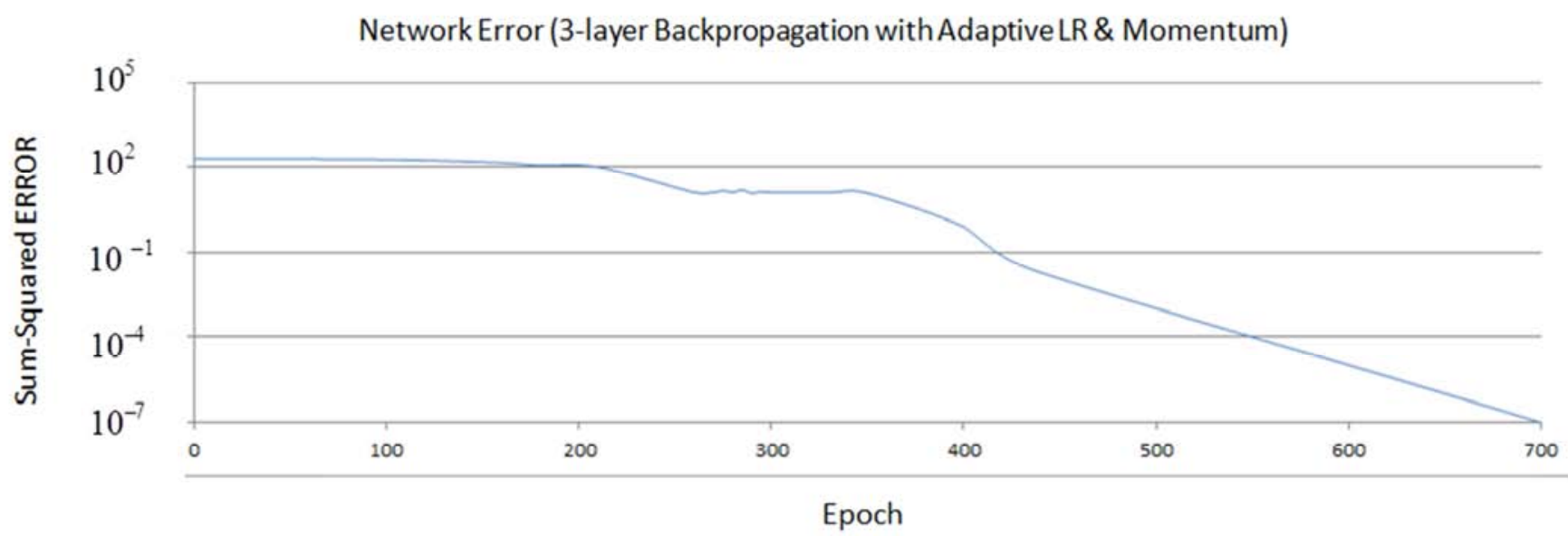

Fig. 6. Sum-squared error.

In the learning fusion method of context-awareness, the fluid property sensor network can get more effective analysis of information processing and response, after the completion of pre-training, since the neural network can be very fast on the learning fusion type even if the classification, the simulation time only takes a few seconds. The universes of discourse connected to the part are

$$
W_{e_{\theta_{p}}}\left(=W_{e_{d_{p}}}=W_{e_{y_{d_{f}}}}=W_{e_{y_{d_{v}}}}\right)=[0, \ldots, 6] \text {. }
$$

The results in Fig. 6 represent the curves for classifying the intermediate operating conditions in the fluid property sensor network with the change measurement of the squared error by the neural network. Through careful study of these data, the results show that the maximum length of $6 \mathrm{~cm}$.

Probability of success and fuzzy entropy at each training step is shown in Fig. 7.

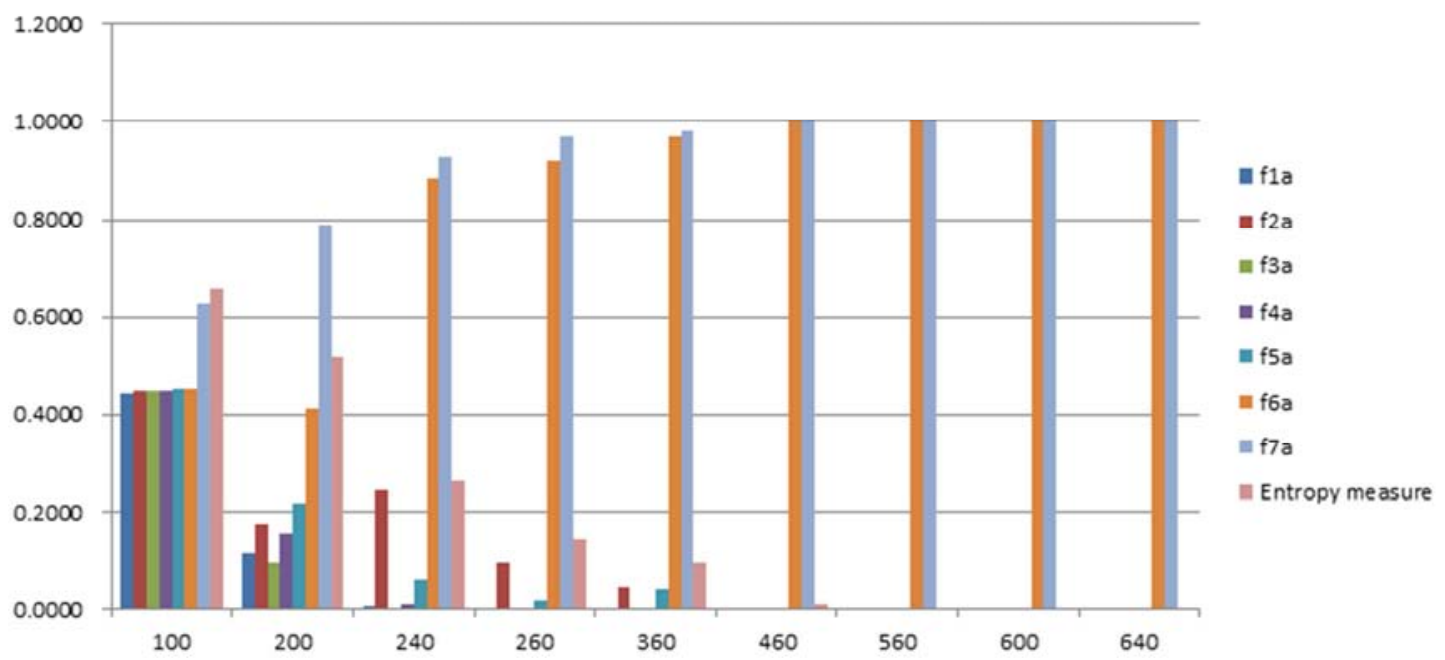

Fig. 7. Probability of success and fuzzy entropy at each training step.

The results in Fig. 7 also show that the proposed method can provide the correct classification for learning fusion to achieve for the context-awareness and learning fusion method. To classify $i_{k}$ will be with the increase in the number of training increased. Learning fusion in a sensor network is actually a process to correctly image the input, and the normalized output is shown in Table 3.
Table 3. Fuzzy entropy measure for feasible plans (macro-learning fusion I).

\begin{tabular}{llc}
\hline Feasible & Order of events & $\begin{array}{c}\text { Entropy } \\
\text { measure }\end{array}$ \\
\hline$p_{a}$ & $e_{\theta_{p}}=49.2^{\circ} \rightarrow e_{d_{p}}=10.6(\mathrm{~cm}) \rightarrow e_{x_{d_{h}}}=4.6(\mathrm{~cm})$ & \\
& $\rightarrow e_{y_{d f}}=7.5(\mathrm{~cm}) \rightarrow e_{\theta_{p}}=45.4^{\circ} \rightarrow e_{d_{p}}=25.6(\mathrm{~cm})$ & 9.760 \\
& $e_{\theta_{p}}=45.4^{\circ} \rightarrow e_{d_{p}}=10.9(\mathrm{~cm}) \rightarrow e_{x_{d_{h}}}=4.3(\mathrm{~cm})$ & \\
$p_{b}$ & $\rightarrow e_{\theta_{p}}=49.4^{\circ} \rightarrow e_{d_{p}}=5.5(\mathrm{~cm}) \rightarrow e_{x_{d_{h}}}=6.0(\mathrm{~cm})$ & 13.634 \\
& $\rightarrow e_{\theta_{p}}=69.1^{\circ} \rightarrow e_{d_{p}}=23.9(\mathrm{~cm})$ & \\
$\ldots$ & $\ldots \ldots$ &
\end{tabular}


In Table 3, the results of fuzzy entropy measurement may be equivalent to the possibility. When the neural network training ends at 240 times, the output value $\Phi_{6}^{240}$ is 0.987 . Then, according to (6) - (9), the results show that the $Y_{240}$ can be written as (11) in section 4 . The probability of the fuzzy set (Fig. 3) is measured to be 1.028 by (12), and its entropy is calculated as 0.363 with (4). But with the training period increased to 640 , The input image pattern $i_{k}$ is fully equivalent to $\Phi_{6}^{d}$. It can be seen from Fig. 7 that the input pattern is considered to be the correlation between the probabilities of the desired output $\Phi_{6}^{d}$.

\subsection{Further Discussion}

Successful autonomous action in the unknown fluid environment is the most efficient method in fluid property sensor network. In the larger control plan, each movement or rotation of the sensor will be referred as an event, and the fuzzy event means that each sensor learning uncertainty involved. In the motion of the fluid property sensor, efficient or optimal processing means entropy minimization of the movement.

Study of the algorithm can be divided into: before and after study, L1, L2, L3 these 3 parts, a performance index $\chi^{i}$ is 1 or 0 if $v\left(\eta^{\mathrm{i}}\right) \neq v\left(\zeta^{\mathrm{i}}\right) . \eta^{\mathrm{i}}$ is a variable used to represent the position of one end of the actuator and a part. $v\left(\eta^{\mathrm{i}}\right)$ is a function of $\eta^{\mathrm{i}} \cdot v\left(\eta^{\mathrm{i}}\right)$ and $v\left(\zeta^{\mathrm{i}}\right)$ depend on the specific application. For example, $v\left(\eta^{\mathrm{i}}\right)$ and $v\left(\zeta^{\mathrm{i}}\right)$ can be equivalent if there is no fluid obstruction on the working path of the end effector. $\lambda$ is a step increment, It will gradually increase. $\vartheta^{\mathrm{i}}$ is the total accrual cost of performing a task. $P_{s}$ represents the success rate of fuzzy events. $Q$ represents the scaling factor, and $\left\|\eta^{1}-\eta^{\mathrm{i}}\right\|$ represents the Euclidean distance. The fuzzy entropy can be measured, as shown in Fig. 8 and Fig. 9.

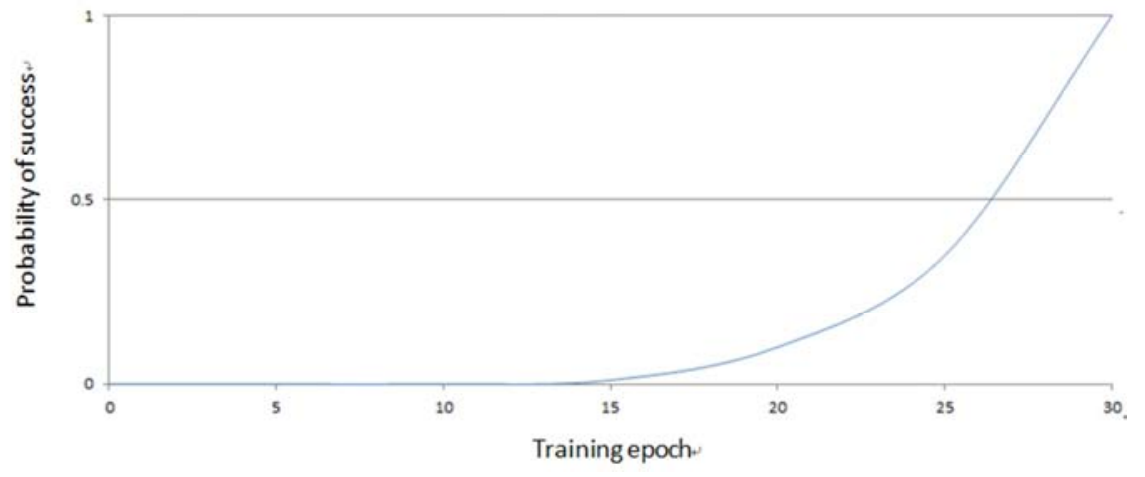

Fig. 8. Probability of success.

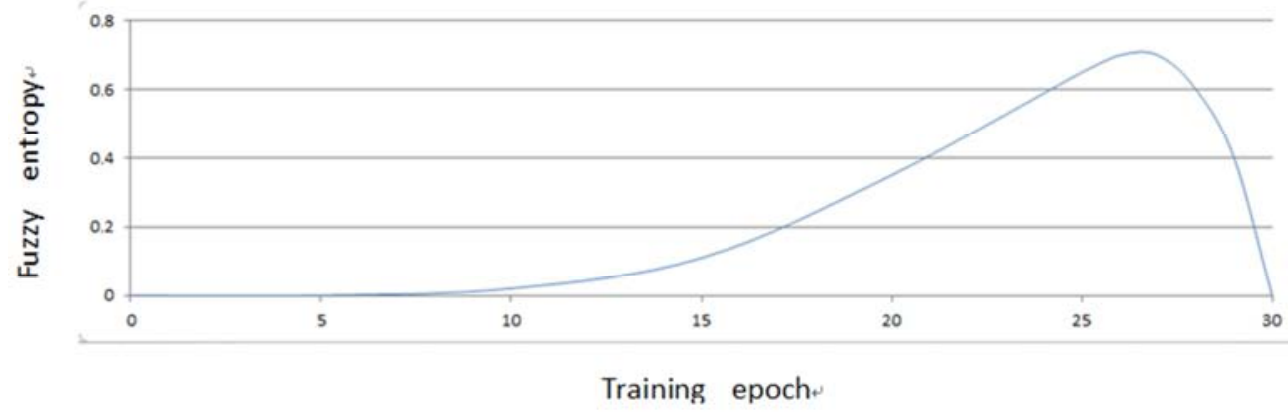

Fig. 9. Fuzzy entropy.

Context-awareness and learning fusion method is used as a criterion for selecting the best plan for a particular task to perform for the fluid property sensor networks. After all feasible plans have been identified, the plan with the lowest degree of uncertainty is selected as the best plan to carry out the task.

In order to deal effectively with a given fluid sensor problem in the fluid property sensor network, the optimal motion of the sensor joint should be achieved, along with the minimum entropy. Fuzzy entropy measure for feasible plans is given in Table 4.
Table 4. Fuzzy entropy measure for feasible plans.

\begin{tabular}{ccc}
\hline Plan & Order of events & Entropy measure \\
\hline$P_{0}$ & $e_{1} e_{2} e_{3} \ldots e_{30} e_{t}$ & 6.758 \\
$P_{1}$ & $e_{1} e_{30} e_{t}$ & 0.208 \\
$P_{2}$ & $e_{2} e_{30} e_{t}$ & 0.209 \\
$P_{3}$ & $e_{3} e_{30} e_{t}$ & 0.200 \\
$\ldots$ & $\ldots$ & $\ldots$ \\
$P_{27}$ & $e_{27} e_{30} e_{t}$ & 0.883 \\
$P_{28}$ & $e_{28} e_{30} e_{t}$ & 0.808 \\
$P_{29}$ & $e_{29} e_{30} e_{t} \mathrm{~S}$ & 0.622 \\
\hline
\end{tabular}


The movement of each part of the fluid property sensor network is defined as an event involving uncertainty. It is therefore necessary to measure the entropy associated with each movement of the fluid property sensor. The lower the uncertainty is, the higher the probability of success. Assuming that the site is initially located at a point, its environment is not strong, and labeled $e_{\mathrm{i}}(i=1, \ldots, 30)$. The distance between $e_{1}$ and $e_{30}$ is $9 \mathrm{~cm}$, the distance between $e_{i}$ and $e_{i+1}$ is $0.3 \mathrm{~cm}$. For illustrative purposes, the set are given as

$$
\begin{gathered}
\left.P_{\mathrm{s}}=\left\{\left(e_{1}, 0.108\right),\left(e_{2}, 0.109\right),\left(e_{3}, 0.101\right)\right\}, \ldots,\left(e_{30}, 0.956\right)\right\}, \\
\left.E[P]=\left\{\left(e_{1}, 0.105\right),\left(e_{2}, 0.104\right),\left(e_{3}, 0.106\right)\right\}, \ldots,\left(e_{30}, 0.522\right)\right\}
\end{gathered}
$$

where $e_{\mathrm{i}}(i=1, \ldots, 30)$, represents 30 starting positions.

Fuzzy entropy can be used to remove unwanted motion in the movement of the sensor from the starting point to the target. In this case, the unnecessary movement can be stopped at each successive point.

In the first run, the total path from point e 1 consists of 30 parts. Each section can be thought of as a test in which an attempt is made to reduce the uncertainty. However, by introducing the learning progresses, the end-effector is finally brought into the state of direct arrival at point e30, i.e., whether the starting point is $e_{\mathrm{i}}(i=1, \ldots, 30)$. Finally, the whole step is reduced to three straight lines. The reduction from 30 paths to only one path is the result of the learning fusion algorithm. This reflects the total accrual entropy can be reduced by learning fusion. In this context, tasks are executed in the order of $e_{1} e_{30} e_{t}$, where the sensor endeffector moves from point $e_{1}$ to point $e_{30}$ to point $e$. The total entropy of the planned $\mathrm{p} 0$ in the master plan is the largest, with a value of 6.758 . On this basis, plan $p 1$ has the lowest degree of uncertainty, the fuzzy entropy measure is 0.208 to be chosen as the best plan to be carried out.

\section{Conclusions}

This paper puts forward a fuzzy entropy method to diminish the uncertain information in fluid property sensor networks. The correlation between learning fusion and context awareness are coincident and correlated, and fuzzy entropy can help make uncertain information fusion. The uncertainty and execution time of information analysis and processing are often conflicted, but can be coincided by the optimized criterion. This paper also introduces a kind of information fusion technology of multiple sensor networks for data analysis and strategy formulation. In practical application, various fluid properties are different but learning algorithm could fuse them to provide us a convenient tool for sensor data processing. If multiple networks or notes are in a workspace, the proposed method can also be used similarly to the method discussed above.

In future work, the work should be applied in real fluid environment to find the corresponding target execution time, and the effect of fluid properties of the sensor on the fluid movement should be further analyzed. Total execution time of learning fusion includes more besides the data analysis of fluid property, mobility. The above method for a fluid sensor fusion should be used in a more uncertain fluid environment to verify its performance, such as material sensing, complex context, different fusion tasks, etc.

\section{Acknowledgment}

This research was supported by the National Natural Science Foundation of China (No. 71471102), and Science and Technology Research Program, Hubei Provincial Department of Education in China (Grant No. D20101203).

\section{References}

[1] Pajares Martinsanz, Gonzalo, Sensors for Fluid Leak Detection, Sensors, 15 (2015) 3830-3833.

[2] Dey, Kajal Kumar; Bhatnagar, Divyanshu; Srivastava, Avanish Kumar, VO2 nanorods for efficient performance in thermal fluids and sensors, Nanoscale, 7 (2015) 6159-6172.

[3] He, Yonglin; Liao, Shenglong; Jia, Hanyu, A Self-Healing Electronic Sensor Based on Thermal-Sensitive Fluids, Advanced Materials,27 (2015) 4622-4627.

[4] Matzeu, Giusy; Florea, Larisa; Diamond, Dermot, Advances in wearable chemical sensor design for monitoring biological fluids, SENSORS And Actuators B-Chemical,2 11 (2015) 403-418.

[5] Amrehn, Sabrina; Wu, Xia; Schumacher, Christian, Photonic crystal-based fluid sensors: Toward practical application, Physica Status Solidi A-Applications And Materials Science, 212 (2015) 1266-1272.

[6] Schultz, Joshua A.; Heinrich, Stephen M.; Josse, Fabien, LateralMode Vibration of Microcantilever-Based Sensors in Viscous Fluids Using Timoshenko Beam Theory, Journal Of Microelectromechanical Systems, 24 (2015) 848-860.

[7] Kulapina, O. I.; Makarova, N. M.; Kulapina, E. G, Potentiometric sensors for the determination of some cephalosporin antibiotics in biological fluids and medicinal preparations, Journal of Analytical Chemistry, 70 (2015) 477-484.

[8] Kamel, Ayman H.; Galal, Hoda R, MIP-Based Biomimetic Sensors for Static and Hydrodynamic Potentiometric Transduction of Sitagliptin in Biological Fluids, International Journal Of Electrochemical Science, 9 (2014) 4361-4373.

[9] Ali, Mohammed; Barman, Koushik; Jasimuddin, Sk, Fluid interface-mediated nanoparticle membrane as an electrochemical sensor, Rsc Advances, 4 (2014) 61404-61408.

[10] Xie, Jun; Li, Decai; Xing, Yansi, Parameters optimization of magnetic fluid micro-pressure sensor, Sensors And Actuators APhysical, 235 (2015) 194-202.

[11] Varma, Sarvesh; Voldman, Joel, A cell-based sensor of fluid shear stress for microfluidics, Lab On A Chip, 15 (2015) 1563-1573.

[12] Li, Jianhua; Wang, Rong; Wang, Jingyuan, Novel magnetic field sensor based on magnetic fluids infiltrated dual-core photonic crystal fibers, Optical Fiber Technology, 20 (2014) 100-105. 
[13] Rosy; Yadav, Saurabh K.; Agrawal, Bharati, Graphene modified Palladium sensor for electrochemical analysis of norepinephrine in pharmaceuticals and biological fluids, Electrochimica Acta, 125 (2014) 622-629.

[14] Fernandes, Luis Andre L.; Azadmehr, Mehdi; Johannessen, Erik A, An Osmotic Pressure Sensor for Monitoring the Level of Hydration in Biological Fluids, Ieee Sensors Journal, 16 (2016) 4331-4337.

[15] Bley, Torsten; Steffensky, Joerg; Mannebach, Horst, Degradation monitoring of aviation hydraulic fluids using non-dispersive infrared sensor systems, Sensors And Actuators B-Chemical, 224 (2016) 539-546.

[16] Tadi, Kiran Kumar; Motghare, Ramani V, Voltammetric Determination of Pindolol in Biological Fluids Using Molecularly Imprinted Polymer Based Biomimetic Sensor, Journal Of The Electrochemical Society, 163 (2016) B286-B292.

[17] Yadav, Saurabh K.; Rosy; Oyama, Munetaka, A Biocompatible Nano Gold Modified Palladium Sensor for Determination of Dopamine in Biological Fluids, Journal Of The Electrochemical Society,161 (2014) H41-H46.

[18] Pattar, Vijay P.; Nandibewoor, Sharanappa T, Polybenzoin Based Sensor for Determination of 2thiouracil in Biological Fluids and Pharmaceutical Formulations, Journal Of The Chinese Chemical Society, 62 (2015) 287-295.

[19] Gupta, Pankaj; Yadav, Saurabh K.; Goyal, Rajendra N, A Sensitive Polymelamine Modified Sensor for the Determination of Lomefloxacin in Biological Fluids, Journal Of The Electrochemical Society, 162 (2015) H86-H92.
[20] Stefan-van Staden, Raluca-Ioana; Moldoveanu, Iuliana, Multimode Sensors Based on Nanostructured Materials for Simultaneous Screening of Biological Fluids for Specific Breast Cancer and Hepatitis B Biomarkers, Journal Of The Electrochemical Society, 161 (2014) B45-B48.

[21] Alarfaj, Nawal A.; El-Tohamy, Maha F, Construction and Validation of New Electrochemical Carbon Nanotubes Sensors for Determination of Acebutolol Hydrochloride in Pharmaceuticals and Biological Fluids, Journal Of The Chinese Chemical Society, 61 (2014) 910-920.

[22] Schmitt, B.; Kiefer, C.; Schuetze, A, Microthermal sensors for determining fluid composition and flow rate in fluidic systems, Microsystem Technologies-Micro-And Nanosystems-Information Storage And Processing Systems, 20 (2014) 641-652.

[23] Couto, Rosa A. S.; Lima, Jose L. F. C.; Quinaz, M. Beatriz, Screen-printed Electrode Based Electrochemical Sensor for the Detection of Isoniazid in Pharmaceutical Formulations and Biological Fluids, International Journal Of Electrochemical Science, 10 (2015) 8738-8749.

[24] Raj, Mamta; Gupta, Pankaj; Goyal, Rajendra N, Poly-Melamine Film Modified Sensor for the Sensitive and Selective Determination of Propranolol, a beta-blocker in Biological Fluids, Journal Of The Electrochemical Society, 163 (2016) H388-H394.

[25] Afkhami, Abbas; Soltani-Felehgari, Farzaneh; Madrakian, Tayyebeh, A sensitive electrochemical sensor for rapid determination of methadone in biological fluids using carbon paste electrode modified with gold nanofilm, Talanta, 128 (2014) 203-210. 